Article

\title{
Slice Holomorphic Functions in Several Variables with Bounded $L$-Index in Direction
}

\author{
Andriy Bandura 1,* (D) and Oleh Skaskiv ${ }^{2}$ (D) \\ 1 Department of Advanced Mathematics, Ivano-Frankivsk National Technical University of Oil and Gas, \\ 76019 Ivano-Frankivsk, Ukraine \\ 2 Department of Mechanics and Mathematics, Ivan Franko National University of Lviv, 79000 Lviv, Ukraine \\ * Correspondence: andriykopanytsia@gmail.com
}

Received: 25 June 2019; Accepted: 25 July 2019; Published: 26 July 2019

check for updates

\begin{abstract}
In this paper, for a given direction $\mathbf{b} \in \mathbb{C}^{n} \backslash\{\boldsymbol{0}\}$ we investigate slice entire functions of several complex variables, i.e., we consider functions which are entire on a complex line $\left\{z^{0}+t \mathbf{b}\right.$ : $t \in \mathbb{C}\}$ for any $z^{0} \in \mathbb{C}^{n}$. Unlike to quaternionic analysis, we fix the direction $\mathbf{b}$. The usage of the term slice entire function is wider than in quaternionic analysis. It does not imply joint holomorphy. For example, it allows consideration of functions which are holomorphic in variable $z_{1}$ and continuous in variable $z_{2}$. For this class of functions there is introduced a concept of boundedness of $L$-index in the direction $\mathbf{b}$ where $\mathbf{L}: \mathbb{C}^{n} \rightarrow \mathbb{R}_{+}$is a positive continuous function. We present necessary and sufficient conditions of boundedness of $L$-index in the direction. In this paper, there are considered local behavior of directional derivatives and maximum modulus on a circle for functions from this class. Also, we show that every slice holomorphic and joint continuous function has bounded $L$-index in direction in any bounded domain and for any continuous function $L: \mathbb{C}^{n} \rightarrow \mathbb{R}_{+}$.
\end{abstract}

Keywords: bounded index; bounded L-index in direction; slice function; entire function; bounded $l$-index

MSC: 32A10; 32A17; 32A37; 30H99; 30A05

\section{Introduction}

In recent years, analytic functions of several variables with bounded index have been intensively investigated. The main objects of investigations are such function classes: entire functions of several variables [1-3], functions analytic in a polydisc [4], in a ball [5] or in the Cartesian product of the complex plane and the unit disc [6].

For entire functions and analytic function in a ball there were proposed two approaches to introduce a concept of index boundedness in a multidimensional complex space. They generate so-called functions of bounded L-index in a direction, and functions of bounded L-index in joint variables.

Let us introduce some notations and definitions.

Let $\mathbb{R}_{+}=(0,+\infty), \mathbb{R}_{+}^{*}=[0,+\infty), \mathbf{0}=(0, \ldots, 0), \mathbf{b}=\left(b_{1}, \ldots, b_{n}\right) \in \mathbb{C}^{n} \backslash\{\mathbf{0}\}$ be a given direction, $L: \mathbb{C}^{n} \rightarrow \mathbb{R}_{+}$be a continuous function, $F: \mathbb{C}^{n} \rightarrow \mathbb{C}$ an entire function. The slice functions on a line $\left\{z^{0}+t \mathbf{b}: t \in \mathbb{C}\right\}$ for fixed $z^{0} \in \mathbb{C}^{n}$ we will denote as $g_{z^{0}}(t)=F\left(z^{0}+t \mathbf{b}\right)$ and $l_{z^{0}}(t)=L\left(z^{0}+t \mathbf{b}\right)$.

Definition 1 ([7]). An entire function $F: \mathbb{C}^{n} \rightarrow \mathbb{C}$ is called a function of bounded L-index in a direction $\mathbf{b}$, if there exists $m_{0} \in \mathbb{Z}_{+}$such that for every $m \in \mathbb{Z}_{+}$and for all $z \in \mathbb{C}^{n}$ one has

$$
\frac{\left|\partial_{\mathbf{b}}^{m} F(z)\right|}{m ! L^{m}(z)} \leq \max _{0 \leq k \leq m_{0}} \frac{\left|\partial_{\mathbf{b}}^{k} F(z)\right|}{k ! L^{k}(z)}
$$


where $\partial_{\mathbf{b}}^{0} F(z)=F(z), \partial_{\mathbf{b}} F(z)=\sum_{j=1}^{n} \frac{\partial F(z)}{\partial z_{j}} b_{j}, \partial_{\mathbf{b}}^{k} F(z)=\partial_{\mathbf{b}}\left(\partial_{\mathbf{b}}^{k-1} F(z)\right), k \geq 2$

The least such integer number $m_{0}$, obeying (1), is called the $L$-index in the direction $\mathbf{b}$ of the function $F$ and is denoted by $N_{\mathbf{b}}(F, L)$. If such $m_{0}$ does not exist, then we put $N_{\mathbf{b}}(F, L)=\infty$, and the function $F$ is said to be of unbounded $L$-index in the direction $\mathbf{b}$ in this case. If $L(z) \equiv 1$, then the function $F$ is said to be of bounded index in the direction $\mathbf{b}$ and $N_{\mathbf{b}}(F)=N_{\mathbf{b}}(F, 1)$ is called the index in the direction $\mathbf{b}$. Let $l: \mathbb{C} \rightarrow \mathbb{R}_{+}$be a continuous function. For $n=1$, $\mathbf{b}=1, L(z) \equiv l(z), z \in \mathbb{C}$ inequality (1) defines a function of bounded $l$-index with the $l$-index $N(F, l) \equiv N_{1}(F, l)[8,9]$, and if in addition $l(z) \equiv 1$, then we obtain a definition of index boundedness with index $N(F) \equiv N_{1}(F, 1)[10,11]$. It is also worth to mention paper [12], which introduces the concept of generalized index. It is quite close to the bounded $l$-index. Let $N_{\mathbf{b}}\left(F, L, z^{0}\right)$ stands for the $L$-index in the direction $\mathbf{b}$ of the function $F$ at the point $z^{0}$, i.e., it is the least integer $m_{0}$, for which inequality (1) is satisfied at this point $z=z^{0}$. By analogy, the notation $N\left(f, l, z^{0}\right)$ is defined if $n=1$, i.e., in the case of functions of one variable.

The concept of $L$-index boundedness in direction requires to consider a slice $\left\{z^{0}+t \mathbf{b}: t \in \mathbb{C}\right\}$. We fixed $z^{0} \in \mathbb{C}^{n}$ and used considerations from one-dimensional case. Then we construct uniform estimates above all $z^{0}$. This is a nutshell of the method.

In view of this, Prof S. Yu. Favorov (2015) posed the following problem in a conversation with one of the authors.

Problem 1 ([13]). Let $\mathbf{b} \in \mathbb{C}^{n} \backslash\{\mathbf{0}\}$ be a given direction, $L: \mathbb{C}^{n} \rightarrow \mathbb{R}_{+}$be a continuous function. Is it possible

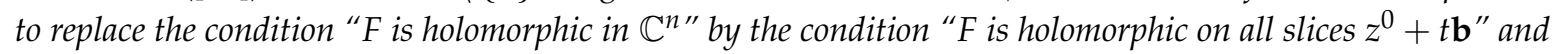
to deduce all known properties of entire functions of bounded L-index in direction for this function class?

There is a negative answer to Favorov's question [13]. This relaxation of restrictions by the function $F$ does not allow the proving of some theorems. Here by $\bar{D}$ we denote a closure of domain $D$. There was proved the following proposition.

Proposition $\mathbf{1}$ ([13], Theorem 5). For every direction $\mathbf{b} \in \mathbb{C}^{n} \backslash\{\mathbf{0}\}$ there exists a function $F(z)$ and a bounded domain $D \subset \mathbb{C}^{n}$ with following properties:

(1) $F$ is holomorphic function of bounded index on every slice $\left\{z^{0}+t \mathbf{b}: t \in \mathbb{C}\right\}$ for each fixed $z^{0} \in \mathbb{C}^{n}$;

(2) $F$ is not entire function in $\mathbb{C}^{n}$;

(3) $F$ does not satisfy (1) in $\bar{D}$, i.e., for any $p \in \mathbb{Z}_{+}$there exists $m \in \mathbb{Z}_{+}$and $z_{p} \in \bar{D}$

$$
\frac{\left|\partial_{\mathbf{b}}^{m} F\left(z_{p}\right)\right|}{m !}>\max \left\{\frac{\left|\partial_{\mathbf{b}}^{k} F\left(z_{p}\right)\right|}{k !}: 0 \leq k \leq p\right\} .
$$

Let $D$ be a bounded domain in $\mathbb{C}^{n}$. If inequality (1) holds for all $z \in D$ instead $\mathbb{C}^{n}$, then $F$ is called function of bounded L-index in the direction $\mathbf{b}$ in the domain $D$. The least such integer $m_{0}$ is called the $L$-index in the direction $\mathbf{b} \in \mathbb{C}^{n} \backslash\{\mathbf{0}\}$ in the domain $D$ and is denoted by $N_{\mathbf{b}}(F, L, D)=m_{0}$.

Proposition 2 ([13], Theorem 2). Let $D$ be a bounded domain in $\mathbb{C}^{n}, \mathbf{b} \in \mathbb{C}^{n} \backslash\{\mathbf{0}\}$ be arbitrary direction. If $L: \mathbb{C}^{n} \rightarrow \mathbb{R}_{+}$is continuous function and $F(z)$ is an entire function such that $\left(\forall z^{0} \in \bar{D}\right): \quad F\left(z^{0}+t \mathbf{b}\right) \not \equiv 0$, then $N_{\mathbf{b}}(F, L, D)<\infty$.

Hence, if we replace holomorphy in $\mathbb{C}^{n}$ by holomorphy on the slices $\left\{z^{0}+t \mathbf{b}: t \in \mathbb{C}\right\}$, then conclusion of Proposition 2 is not valid. Thus, Proposition 1 shows impossibility to replace joint holomorphy by slice holomorphy without additional hypothesis. The proof of Proposition 2 uses 
continuity in joint variables (see [13], Equation (6)). It leads to the following question (see [14], where it is also formulated. There was considered a case $L(z) \equiv 1)$.

Problem 2. What are additional conditions providing validity of Proposition 2 for slice holomorphic functions?

A main goal of this investigation is to deduce an analog of Proposition 2 for slice holomorphic functions.

Please note that the positivity and continuity of the function $L$ are weak restrictions to deduce constructive results. Thus, we assume additional restrictions by the function $L$.

Let us denote

$$
\lambda_{\mathbf{b}}(\eta)=\sup _{z \in \mathbb{C}^{n}} \sup _{t_{1}, t_{2} \in \mathbb{C}}\left\{\frac{L\left(z+t_{1} \mathbf{b}\right)}{L\left(z+t_{2} \mathbf{b}\right)}:\left|t_{1}-t_{2}\right| \leq \frac{\eta}{\min \left\{L\left(z+t_{1} \mathbf{b}\right), L\left(z+t_{2} \mathbf{b}\right)\right\}}\right\} .
$$

By $Q_{\mathbf{b}}^{n}$ we denote a class of positive continuous function $L: \mathbb{C}^{n} \rightarrow \mathbb{R}_{+}$, satisfying the condition

$$
(\forall \eta \geq 0): \lambda_{\mathbf{b}}(\eta)<+\infty
$$

Moreover, it is sufficient to require validity of (2) for one value $\eta>0$.

For a positive continuous function $l(t), t \in \mathbb{C}$, and $\eta>0$ we define $\lambda(\eta) \equiv \lambda_{1}^{\mathbf{b}}(\eta)$ in the cases when $\mathbf{b}=1, n=1, L \equiv l$. As in [15], let $Q \equiv Q_{1}^{1}$ be a class of positive continuous functions $l(t), t \in \mathbb{C}$, obeying the condition $0<\lambda(\eta)<+\infty$ for all $\eta>0$.

Besides, we denote by $\langle a, c\rangle=\sum_{j=1}^{n} a_{j} \overline{c_{j}}$ the scalar product in $\mathbb{C}^{n}$, where $a, c \in \mathbb{C}^{n}$.

Let $\widetilde{\mathcal{H}}_{\mathbf{b}}^{n}$ be a class of functions which are holomorphic on every slices $\left\{z^{0}+t \mathbf{b}: t \in \mathbb{C}\right\}$ for each $z^{0} \in \mathbb{C}^{n}$ and let $\mathcal{H}_{\mathbf{b}}^{n}$ be a class of functions from $\widetilde{\mathcal{H}}_{\mathbf{b}}^{n}$ which are joint continuous. The notation $\partial_{\mathbf{b}} F(z)$ stands for the derivative of the function $g_{z}(t)$ at the point 0 , i.e., for every $p \in \mathbb{N} \quad \partial_{\mathbf{b}}^{p} F(z)=g_{z}^{(p)}(0)$, where $g_{z}(t)=F(z+t \mathbf{b})$ is entire function of complex variable $t \in \mathbb{C}$ for given $z \in \mathbb{C}^{n}$. In this research, we will often call this derivative as directional derivative because if $F$ is entire function in $\mathbb{C}^{n}$ then the derivatives of the function $g_{z}(t)$ matches with directional derivatives of the function $F$.

Please note that if $F \in \mathcal{H}_{\mathbf{b}}^{n}$ then for every $p \in \mathbb{N} \partial_{\mathbf{b}} F \in \mathcal{H}_{\mathbf{b}}^{n}$. It can be proved by using of Cauchy's formula.

Together the hypothesis on joint continuity and the hypothesis on holomorphy in one direction do not imply holomorphy in whole $n$-dimensional complex space. We give some examples to demonstrate it. For $n=2$ let $f: \mathbb{C} \rightarrow \mathbb{C}$ be an entire function, $g: \mathbb{C} \rightarrow \mathbb{C}$ be a continuous function. Then $f\left(z_{1}\right) g\left(z_{2}\right)$, $f\left(z_{1}\right) \pm g\left(z_{2}\right), f\left(z_{1} \cdot g\left(z_{2}\right)\right)$ are functions which are holomorphic in the direction $(1,0)$ and are joint continuous in $\mathbb{C}^{2}$. Moreover, if we have performed an affine transformation

$$
\left\{\begin{array}{l}
z_{1}=b_{2} z_{1}^{\prime}+b_{1} z_{2}^{\prime} \\
z_{2}=b_{2} z_{1}^{\prime}-b_{1} z_{2}^{\prime}
\end{array}\right.
$$

then the appropriate new functions are also holomorphic in the direction $\left(b_{1}, b_{2}\right)$ and are joint continuous in $\mathbb{C}^{2}$, where $b_{1} \neq 0, b_{2} \neq 0$.

A function $F \in \widetilde{\mathcal{H}}_{\mathbf{b}}^{n}$ is said to be of bounded L-index in the direction $\mathbf{b}$, if there exists $m_{0} \in \mathbb{Z}_{+}$such that for all $m \in \mathbb{Z}_{+}$and each $z \in \mathbb{C}^{n}$ inequality (1) is true. All notations, introduced above for entire functions of bounded $L$-index in direction, keep for functions from $\widetilde{\mathcal{H}}_{\mathbf{b}}^{n}$.

\section{Sufficient Sets}

Now we prove several assertions that establish a connection between functions of bounded $L$-index in direction and functions of bounded $l$-index of one variable. The similar results for entire functions of several variables were obtained in $[7,16]$. The next proofs use ideas from the mentioned 
papers. The proofs of Propositions 3, 4 and Theorems 1, 2 literally repeat arguments from proofs of corresponding propositions for entire functions $[7,16]$. Therefore, we omit these proofs.

Proposition 3. If a function $F \in \widetilde{\mathcal{H}}_{\mathbf{b}}^{n}$ has bounded L-index in the direction $\mathbf{b}$ then for every $z^{0} \in \mathbb{C}^{n}$ the entire function $g_{z^{0}}(t)$ is of bounded $l_{z^{0}}$-index and $N\left(g_{z^{0}}, l_{z^{0}}\right) \leq N_{\mathbf{b}}(F, L)$.

Proposition 4. If a function $F \in \widetilde{\mathcal{H}}_{\mathbf{b}}^{n}$ has bounded L-index in the direction $\mathbf{b}$ then

$$
N_{\mathbf{b}}(F, L)=\max \left\{N\left(g_{z^{0}}, l_{z^{0}}\right): z^{0} \in \mathbb{C}^{n}\right\} .
$$

Theorem 1. A function $F \in \widetilde{\mathcal{H}}_{\mathbf{b}}^{n}$ has bounded L-index in the direction $\mathbf{b}$ if and only if there exists a number $M>0$ such that for all $z^{0} \in \mathbb{C}^{n}$ the function $g_{z^{0}}(t)$ is of bounded $l_{z^{0}}$-index with $N\left(g_{z^{0}}, l_{z^{0}}\right) \leq M<+\infty$, as a function of variable $t \in \mathbb{C}$. Thus, $N_{\mathbf{b}}(F, L)=\max \left\{N\left(g_{z^{0}}, l_{z^{0}}\right): z^{0} \in \mathbb{C}^{n}\right\}$.

Theorem 2. Let $\mathbf{b} \in \mathbb{C}^{n} \backslash\{\mathbf{0}\}$ be a given direction, $A_{0} \subset \mathbb{C}^{n}$ such that $\left\{z+t \mathbf{b}: t \in \mathbb{C}, z \in A_{0}\right\}=\mathbb{C}^{n}$. A function $F \in \widetilde{\mathcal{H}}_{\mathbf{b}}^{n}$ has bounded $L$-index in the direction $\mathbf{b}$ if and only if there exists a number $M>0$ such that for all $z^{0} \in A_{0}$ the function $g_{z^{0}}(t)$ is of bounded $l_{z^{0}}$-index with $N\left(g_{z^{0}}, l_{z^{0}}\right) \leq M<+\infty$, as a function of one variable $t \in \mathbb{C}$ and $N_{\mathbf{b}}(F, L)=\max \left\{N\left(g_{z^{0}}, l_{z^{0}}\right): z^{0} \in A_{0}\right\}$.

Remark 1. An arbitrary hyperplane $A_{0}=\left\{\tilde{z} \in \mathbb{C}^{n}:\langle\tilde{z}, c\rangle=1\right\}$, where $\langle c, \mathbf{b}\rangle \neq 0$, satisfies conditions of Theorem 2.

Corollary 1. If $F \in \widetilde{\mathcal{H}}_{\mathbf{b}}^{n}$ is of bounded L-index in the direction $\mathbf{b}$ and $j_{0}$ is chosen such that $b_{j_{0}} \neq 0$, then $N_{\mathbf{b}}(F, L)=\max \left\{N\left(g_{z^{0}}, l_{z^{0}}\right): z^{0} \in \mathbb{C}^{n}, z_{j_{0}}^{0}=0\right\}$, and if $\sum_{j=0}^{n} b_{j} \neq 0$, then $N_{\mathbf{b}}(F, L)=\max \left\{N\left(g_{z^{0}}, l_{z^{0}}\right)\right.$ : $\left.z^{0} \in \mathbb{C}^{n}, \sum_{j=0}^{n} z_{j}^{0}=0\right\}$

We note that for a given $z \in \mathbb{C}^{n}$ the choice of $z^{0} \in \mathbb{C}^{n}$ and $t \in \mathbb{C}$ such that $\sum_{j=1}^{n} z_{j}^{0}=0$ and $z=z^{0}+t \mathbf{b}$, is unique.

Theorem 3 requires replacement of the space $\widetilde{\mathcal{H}}_{\mathbf{b}}^{n}$ by the space $\mathcal{H}_{\mathbf{b}}^{n}$. In other words, we use joint continuity in its proof.

Theorem 3. Let $\bar{A}=\mathbb{C}^{n}$, i.e., $A$ be an everywhere dense set in $\mathbb{C}^{n}$ and let a function $F \in \mathcal{H}_{\mathbf{b}}^{n}$. The function $F$ is of bounded L-index in the direction $\mathbf{b}$ if and only if there exists $M>0$ such that for all $z^{0} \in A$ a function $g_{z^{0}}(t)$ is of bounded $l_{z^{0}}$-index $N\left(g_{z^{0}}, l_{z^{0}}\right) \leq M<+\infty$ and $N_{\mathbf{b}}(F, L)=\max \left\{N\left(g_{z^{0}}, l_{z^{0}}\right): z^{0} \in A\right\}$.

Proof. The necessity follows from Theorem 1.

Sufficiency. Since $\bar{A}=\mathbb{C}^{n}$, then for every $z^{0} \in \mathbb{C}^{n}$ there exists a sequence $z^{(m)}$, that $z^{(m)} \rightarrow z^{0}$ as $m \rightarrow+\infty$ and $z^{(m)} \in A$ for all $m \in \mathbb{N}$. However, $F(z+t \mathbf{b})$ is of bounded $l_{z}$-index for all $z \in \bar{A}$ as a function of variable $t$. That is why in view the definition of bounded $l_{z}$-index there exists $M>0$ that

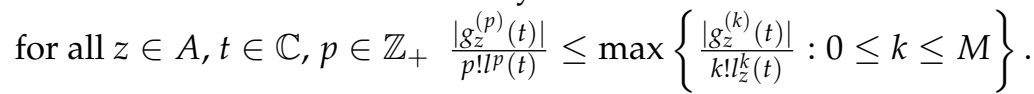

Substituting instead of $z$ a sequence $z^{(m)} \in A, z^{(m)} \rightarrow z^{0}$, we obtain that for every $m \in \mathbb{N}$

$$
\frac{\left|\partial_{\mathbf{b}}^{p} F\left(z^{(m)}+t \mathbf{b}\right)\right|}{p ! L^{p}\left(z^{(m)}+t \mathbf{b}\right)} \leq \max \left\{\frac{\left|\partial_{\mathbf{b}}^{k} F\left(z^{(m)}+t \mathbf{b}\right)\right|}{k ! L^{k}\left(z^{(m)}+t \mathbf{b}\right)}: 0 \leq k \leq M\right\} .
$$


However, $F$ and $\partial_{\mathbf{b}}^{p} F$ are continuous in $\mathbb{C}^{n}$ for all $p \in \mathbb{N}$ and $L$ is a positive continuous function. Thus, in the obtained expression the limiting transition is possible as $m \rightarrow+\infty\left(z^{(m)} \rightarrow z^{0}\right)$. Evaluating the limit as $m \rightarrow+\infty$ we obtain that for all $z^{0} \in \mathbb{C}^{n}, t \in \mathbb{C}, m \in \mathbb{Z}_{+}$

$$
\frac{\left|\partial_{\mathbf{b}}^{p} F\left(z^{0}+t \mathbf{b}\right)\right|}{p ! L^{p}\left(z^{0}+t \mathbf{b}\right)} \leq \max \left\{\frac{\left|\partial_{\mathbf{b}}^{k} F\left(z^{0}+t \mathbf{b}\right)\right|}{k ! L^{k}\left(z^{0}+t \mathbf{b}\right)}: 0 \leq k \leq M\right\} .
$$

This inequality implies that $F(z+t \mathbf{b})$ is of bounded $L(z+t \mathbf{b})$-index as a function of variable $t$ for every given $z \in \mathbb{C}^{n}$. Applying Theorem 1 we obtain the desired conclusion. Theorem 3 is proved.

Remark 1 and Theorem 3 imply the following corollary.

Corollary 2. Let $\mathbf{b} \in \mathbb{C}^{n} \backslash\{\mathbf{0}\}$ be a given direction, $A_{0} \subset \mathbb{C}^{n}$ such that its closure $\bar{A}_{0}=\left\{z \in \mathbb{C}^{n}\right.$ : $\langle z, c\rangle=1\}$, where $\langle c, \mathbf{b}\rangle \neq 0$. And let a function $F \in \mathcal{H}_{\mathbf{b}}^{n}$ and its derivatives $\partial_{\mathbf{b}}^{p} F \in \mathcal{H}_{\mathbf{b}}^{n}$ for all $p \in \mathbb{N}$. The function $F(z)$ is of bounded L-index in the direction $\mathbf{b}$ if and only if there exists a number $M>0$ such that for all $z^{0} \in A_{0}$ the function $g_{z^{0}}(t)$ is of bounded $l_{z^{0}}$ index with $N\left(g_{z^{0}}, l_{z^{0}}\right) \leq M<+\infty$ and $N_{\mathbf{b}}(F, L)=\max \left\{N\left(g_{z^{0}}, l_{z^{0}}\right): z^{0} \in A_{0}\right\}$.

\section{Local Behavior of Directional Derivative}

The following proposition is crucial in theory of functions of bounded index. It initializes series of propositions which are necessary to prove logarithmic criterion of index boundedness. It was first obtained by G. H. Fricke [17] for entire functions of bounded index. Later the proposition was generalized for entire functions of bounded $l$-index [18], analytic functions of bounded $l$-index [19], entire functions of bounded $L$-index in direction [7], functions analytic in a polydisc [4] or in a ball [5] with bounded L-index in joint variables,

Theorem 4. Let $L \in Q_{\mathbf{b}}^{n}$. A function $F \in \widetilde{\mathcal{H}}_{\mathbf{b}}^{n}$ is of bounded L-index in the direction $\mathbf{b}$ if and only if for each $\eta>0$ there exist $n_{0}=n_{0}(\eta) \in \mathbb{Z}_{+}$and $P_{1}=P_{1}(\eta) \geq 1$ such that for every $z \in \mathbb{C}^{n}$ there exists $k_{0}=k_{0}(z) \in \mathbb{Z}_{+}, 0 \leq k_{0} \leq n_{0}$, and

$$
\max \left\{\left|\partial_{\mathbf{b}}^{k_{0}} F(z+t \mathbf{b})\right|:|t| \leq \frac{\eta}{L(z)}\right\} \leq P_{1}\left|\partial_{\mathbf{b}}^{k_{0}} F(z)\right| .
$$

Proof. Our proof is based on the proof of appropriate theorem for entire functions of bounded $L$-index in direction [7].

Necessity. Let $N_{\mathbf{b}}(F ; L) \equiv N<+\infty$. Let $[a], a \in \mathbb{R}$, stands for the integer part of the number $a$ in this proof. We denote

$$
q(\eta)=\left[2 \eta(N+1)\left(\lambda_{\mathbf{b}}(\eta)\right)^{2 N+1}\right]+1 .
$$

For $z \in \mathbb{C}^{n}$ and $p \in\{0,1, \ldots, q(\eta)\}$ we put

$$
\begin{aligned}
& R_{p}^{\mathbf{b}}(z, \eta)=\max \left\{\frac{\left|\partial_{\mathbf{b}}^{k} F(z+t \mathbf{b})\right|}{k ! L^{k}(z+t \mathbf{b})}:|t| \leq \frac{p \eta}{q(\eta) L(z)}, 0 \leq k \leq N\right\}, \\
& \widetilde{R}_{p}^{\mathbf{b}}(z, \eta)=\max \left\{\frac{\left|\partial_{\mathbf{b}}^{k} F(z+t \mathbf{b})\right|}{k ! L^{k}(z)}:|t| \leq \frac{p \eta}{q(\eta) L(z)}, 0 \leq k \leq N\right\} .
\end{aligned}
$$


However, $|t| \leq \frac{p \eta}{q(\eta) L(z)} \leq \frac{\eta}{L(z)}$, then $\lambda_{\mathbf{b}}\left(\frac{p \eta}{q(\eta)}\right) \leq \lambda_{\mathbf{b}}(\eta)$. It is clear that $R_{p}^{\mathbf{b}}(z, \eta), \widetilde{R}_{p}^{\mathbf{b}}(z, \eta)$ are well-defined. Moreover,

$$
\begin{gathered}
R_{p}^{\mathbf{b}}(z, \eta)= \\
=\max \left\{\frac{\left|\partial_{\mathbf{b}}^{k} F(z+t \mathbf{b})\right|}{k ! L^{k}(z)}\left(\frac{L(z)}{L(z+t \mathbf{b})}\right)^{k}: 0 \leq k \leq N,|t| \leq \frac{p \eta}{q(\eta) L(z)}\right\} \leq \\
\leq \max \left\{\frac{\left|\partial_{\mathbf{b}}^{k} F(z+t \mathbf{b})\right|}{k ! L^{k}(z)}\left(\lambda_{\mathbf{b}}\left(\frac{p \eta}{q(\eta)}\right)\right)^{k}:|t| \leq \frac{p \eta}{q(\eta) L(z)}, 0 \leq k \leq N\right\} \leq \\
\leq \max \left\{\frac{\left|\partial_{\mathbf{b}}^{k} F(z+t \mathbf{b})\right|}{k ! L^{k}(z)}\left(\lambda_{\mathbf{b}}(\eta)\right)^{k}:|t| \leq \frac{p \eta}{q(\eta) L(z)}, 0 \leq k \leq N\right\} \leq \\
\leq\left(\lambda_{\mathbf{b}}(\eta)\right)^{N} \max \left\{\frac{\left|\partial_{\mathbf{b}}^{k} F(z+t \mathbf{b})\right|}{k ! L^{k}(z)}:|t| \leq \frac{p \eta}{q(\eta) L(z)}, 0 \leq k \leq N\right\}= \\
=\widetilde{R}_{p}^{\mathbf{b}}(z, \eta)\left(\lambda_{\mathbf{b}}(\eta)\right)^{N}, \\
=\max \left\{\frac{\left|\partial_{\mathbf{b}}^{k} F(z+t \mathbf{b})\right|}{k ! L^{k}(z+t \mathbf{b})}\left(\frac{L(z+t \mathbf{b})}{L(z)}\right)^{k}:|t| \leq \frac{p \eta}{q(\eta) L(z)}, 0 \leq k \leq N\right\} \leq \\
\leq \max \left\{\frac{\left|\partial_{\mathbf{b}}^{k} F(z+t \mathbf{b})\right|}{k ! L^{k}(z+t \mathbf{b})}\left(\lambda_{\mathbf{b}}\left(\frac{p \eta}{q(\eta)}\right)\right)^{k}:|t| \leq \frac{p \eta}{q(\eta) L(z)}, 0 \leq k \leq N\right\} \leq \\
\leq \max \left\{\left(\lambda_{\mathbf{b}}(\eta)\right)^{k} \frac{\left|\partial_{\mathbf{b}}^{k} F(z+t \mathbf{b})\right|}{k ! L^{k}(z+t \mathbf{b})}:|t| \leq \frac{p \eta}{q(\eta) L(z)}, 0 \leq k \leq N\right\} \leq \\
\leq\left(\lambda_{\mathbf{b}}(\eta)\right)^{N} \max \left\{\frac{\left|\partial_{\mathbf{b}}^{k} F(z+t \mathbf{b})\right|}{k ! L^{k}(z+t \mathbf{b})}:|t| \leq \frac{p \eta}{q(\eta) L(z)}, 0 \leq k \leq N\right\}= \\
=R R_{p}^{\mathbf{b}}(z, \eta)\left(\lambda_{\mathbf{b}}(\eta)\right)^{N} .
\end{gathered}
$$

Let $k_{p}^{z} \in \mathbb{Z}, 0 \leq k_{p}^{z} \leq N$, and $t_{p}^{z} \in \mathbb{C},\left|t_{p}^{z}\right| \leq \frac{p \eta}{q(\eta) L(z)}$, be such that

$$
\widetilde{R}_{p}^{\mathbf{b}}(z, \eta)=\frac{\left|\partial_{\mathbf{b}}^{k_{p}^{z}} F\left(z+t_{p}^{z} \mathbf{b}\right)\right|}{k_{p}^{z} ! L^{k_{p}^{z}}(z)} .
$$

However, for every given $z \in \mathbb{C}^{n}$ the function $F(z+t \mathbf{b})$ and its derivative are entire as functions of variables $t$. Then by the maximum modulus principle, equality (6) holds for $t_{p}^{z}$ such that $\left|t_{p}^{z}\right|=\frac{p \eta}{q(\eta) L(z)}$. We set $\widetilde{t_{p}^{z}}=\frac{p-1}{p} t_{p}^{z}$. Then

$$
\begin{gathered}
\left|\widetilde{t_{p}^{z}}\right|=\frac{(p-1) \eta}{q(\eta) L(z)} \\
\left|\widetilde{t_{p}^{z}}-t_{p}^{z}\right|=\frac{\left|t_{p}^{z}\right|}{p}=\frac{\eta}{q(\eta) L(z)} .
\end{gathered}
$$

It follows from (7) and the definition of $\widetilde{R}_{p-1}^{\mathbf{b}}(z, \eta)$ that

$$
\widetilde{R}_{p-1}^{\mathbf{b}}(z, \eta) \geq \frac{\left|\partial_{\mathbf{b}}^{k_{p}^{z}} F\left(z+\widetilde{t}_{p}^{z} \mathbf{b}\right)\right|}{k_{p}^{z} ! L^{k_{p}^{z}}(z)} .
$$

Therefore,

$$
\begin{aligned}
0 & \leq \widetilde{R}_{p}^{\mathbf{b}}(z, \eta)-\widetilde{R}_{p-1}^{\mathbf{b}}(z, \eta) \leq \frac{\left|\partial_{\mathbf{b}}^{k_{p}^{z}} F\left(z+t_{p}^{z} \mathbf{b}\right)\right|-\left|\partial_{\mathbf{b}}^{k_{p}^{z}} F\left(z+\widetilde{t}_{p}^{z} \mathbf{b}\right)\right|}{k_{p}^{z} ! L^{k_{p}^{z}}(z)}= \\
& =\frac{1}{k_{p}^{z} ! L^{k_{p}^{z}}(z)} \int_{0}^{1} \frac{d}{d s}\left|\partial_{\mathbf{b}}^{k_{p}^{z}} F\left(z+\left(\widetilde{t_{p}^{z}}+s\left(t_{p}^{z}-\widetilde{t}_{p}^{z}\right)\right) \mathbf{b}\right)\right| d s .
\end{aligned}
$$


For every analytic complex-valued function of real variable $\varphi(s), s \in \mathbb{R}$, the inequality $\frac{d}{d s}|\varphi(s)| \leq\left|\frac{d}{d s} \varphi(s)\right|$ holds, where $\varphi(s) \neq 0$. Applying this inequality to (9) and using the mean value theorem we obtain

$$
\begin{gathered}
\widetilde{R}_{p}^{\mathbf{b}}\left(z, t_{0}, \eta\right)-\widetilde{R}_{p-1}^{\mathbf{b}}\left(z, t_{0}, \eta\right) \leq \\
\leq \frac{\left|t_{p}^{z}-\widetilde{t}_{p}^{z}\right|}{k_{p}^{z} ! L^{k_{p}^{z}}(z)} \int_{0}^{1}\left|\partial_{\mathbf{b}}^{k_{p}^{z}+1} F\left(z+\left(\widetilde{t}_{p}^{z}+s\left(t_{p}^{z}-\widetilde{t}_{p}^{z}\right)\right) \mathbf{b}\right)\right| d s= \\
=\frac{\left|t_{p}^{z}-\widetilde{t}_{p}^{z}\right|}{k_{p}^{z} ! L^{k_{p}^{z}}(z)}\left|\partial_{\mathbf{b}}^{k_{p}^{z}+1} F\left(z+\left(\widetilde{t}_{p}^{z}+s^{*}\left(t_{p}^{z}-\widetilde{t}_{p}^{z}\right)\right) \mathbf{b}\right)\right|= \\
=L(z)\left(k_{p}^{z}+1\right)\left|t_{p}^{z}-\widetilde{t}_{p}^{z}\right| \frac{\left|\partial_{\mathbf{b}}^{k_{p}^{z}+1} F\left(z+\left(\widetilde{t}_{p}^{z}+s^{*}\left(t_{p}^{z}-\widetilde{t}_{p}^{z}\right)\right) \mathbf{b}\right)\right|}{\left(k_{p}^{z}+1\right) ! L^{k_{p}^{z}+1}(z)},
\end{gathered}
$$

where $s^{*} \in[0,1]$. The point $\widetilde{t}_{p}^{z}+s^{*}\left(t_{p}^{z}-\widetilde{t}_{p}^{z}\right)$ belongs to the set

$$
\left\{t \in \mathbb{C}:|t| \leq \frac{p \eta}{q(\eta) L(z)} \leq \frac{\eta}{L(z)}\right\}
$$

Using the definition of boundedness of $L$-index in direction, the definition of $q(\eta)$, inequalities (4) and (8), for $k_{p}^{z} \leq N$ we have

$$
\begin{gathered}
\widetilde{R}_{p}^{\mathbf{b}}(z, \eta)-\widetilde{R}_{p-1}^{\mathbf{b}}(z, \eta) \leq \frac{\left|\partial_{\mathbf{b}}^{k_{p}^{z}+1} F\left(z+\left(\widetilde{t}_{p}^{z}+s^{*}\left(t_{p}^{z}-\widetilde{t}_{p}^{z}\right)\right) \mathbf{b}\right)\right|}{\left.\left(k_{p}^{z}+1\right) ! L^{k_{p}^{z}+1}\left(z+\widetilde{t}_{p}^{z}+s^{*}\left(t_{p}^{z}-\widetilde{t}_{p}^{z}\right)\right) \mathbf{b}\right)} \times \\
\times\left(\frac{L\left(z+\left(\widetilde{t_{p}^{z}}+s^{*}\left(t_{p}^{z}-\widetilde{t}_{p}^{z}\right)\right) \mathbf{b}\right)}{L(z)}\right)^{k_{p}^{z}+1} L(z)\left(k_{p}^{z}+1\right)\left|t_{p}^{z}-\widetilde{t}_{p}^{z}\right| \leq \eta \frac{N+1}{q(\eta)}\left(\lambda_{\mathbf{b}}(\eta)\right)^{N+1} \times \\
\times \max \left\{\frac{\left|\partial_{\mathbf{b}}^{k} F\left(z+\left(\widetilde{t}_{p}^{z}+s^{*}\left(t_{p}^{z}-\widetilde{t}_{p}^{z}\right)\right) \mathbf{b}\right)\right|}{\left.k ! L^{k}\left(z+\widetilde{t}_{p}^{z}+s^{*}\left(t_{p}^{z}-\widetilde{t}_{p}^{z}\right)\right) \mathbf{b}\right)}: 0 \leq k \leq N\right\} \leq \eta \frac{N+1}{q(\eta)}\left(\lambda_{\mathbf{b}}(\eta)\right)^{N+1} R_{p}^{\mathbf{b}}(z, \eta) \leq \\
\leq \frac{\eta(N+1)\left(\lambda_{\mathbf{b}}(\eta)\right)^{2 N+1}}{\left[2 \eta(N+1)\left(\lambda_{\mathbf{b}}(\eta)\right)^{2 N+1}\right]+1} \widetilde{R}_{p}^{\mathbf{b}}(z, \eta) \leq \frac{1}{2} \widetilde{R}_{p}^{\mathbf{b}}(z, \eta)
\end{gathered}
$$

It follows that $\widetilde{R}_{p}^{\mathbf{b}}(z, \eta) \leq 2 \widetilde{R}_{p-1}^{\mathbf{b}}(z, \eta)$. Using inequalities (4) and (5), we obtain for $R_{p}^{\mathbf{b}}(z, \eta)$

$$
R_{p}^{\mathbf{b}}(z, \eta) \leq 2\left(\lambda_{\mathbf{b}}(\eta)\right)^{N} \widetilde{R}_{p-1}^{\mathbf{b}}(z, \eta) \leq 2\left(\lambda_{\mathbf{b}}(\eta)\right)^{2 N} R_{p-1}^{\mathbf{b}}(z, \eta) .
$$

Hence,

$$
\begin{gathered}
\max \left\{\frac{\left|\partial_{\mathbf{b}}^{k} F(z+t \mathbf{b})\right|}{k ! L^{k}(z+t \mathbf{b})}:|t| \leq \frac{\eta}{L(z)}, 0 \leq k \leq N\right\}=R_{q(\eta)}^{\mathbf{b}}(z, \eta) \leq \\
\leq 2\left(\lambda_{\mathbf{b}}(\eta)\right)^{2 N} R_{q(\eta)-1}^{\mathbf{b}}(z, \eta) \leq\left(2\left(\lambda_{\mathbf{b}}(\eta)\right)^{2 N}\right)^{2} R_{q(\eta)-2}^{\mathbf{b}}(z, \eta) \leq \\
\leq \cdots \leq\left(2\left(\lambda_{\mathbf{b}}(\eta)\right)^{2 N}\right)^{q(\eta)} R_{0}^{\mathbf{b}}(z, \eta)= \\
=\left(2\left(\lambda_{\mathbf{b}}(\eta)\right)^{2 N}\right)^{q(\eta)} \max \left\{\frac{\left|b_{\mathbf{b}}^{k} F(z)\right|}{k ! L^{k}(z)}: 0 \leq k \leq N\right\} .
\end{gathered}
$$

Let $k_{z} \in \mathbb{Z}, 0 \leq k_{z} \leq N$, and $\widetilde{t_{z}} \in \mathbb{C},\left|\widetilde{t_{z}}\right|=\frac{\eta}{L(z)}$ be such that

$$
\frac{\left|\partial_{\mathbf{b}}^{k_{z}} F(z)\right|}{k_{z} ! L^{k_{z}}(z)}=\max _{0 \leq k \leq N} \frac{\left|\partial_{\mathbf{b}}^{k} F(z)\right|}{k ! L^{k}(z)},
$$

and

$$
\left|\partial_{\mathbf{b}}^{k_{z}} F\left(z+\widetilde{t}_{z} \mathbf{b}\right)\right|=\max \left\{\left|\partial_{\mathbf{b}}^{k_{z}} F(z+t \mathbf{b})\right|:|t| \leq \eta / L(z)\right\} .
$$


Inequality (10) implies

$$
\begin{gathered}
\frac{\left|\partial_{\mathbf{b}}^{k_{z}} F\left(z+\widetilde{t_{z}} \mathbf{b}\right)\right|}{k_{z} ! L^{k_{z}}\left(z+\widetilde{t_{z}} \mathbf{b}\right)} \leq \max \left\{\frac{\left|\partial_{\mathbf{b}}^{k_{z}} F(z+t \mathbf{b})\right|}{k_{z} ! L^{k_{z}}(z+t \mathbf{b})}:|t|=\frac{\eta}{L(z)}\right\} \leq \\
\leq \max \left\{\frac{\left|\partial_{\mathbf{b}}^{k} F(z+t \mathbf{b})\right|}{k ! L^{k}(z+t \mathbf{b})}:|t|=\frac{\eta}{L(z)}, 0 \leq k \leq N\right\} \leq \\
\leq\left(2\left(\lambda_{\mathbf{b}}(\eta)\right)^{2 N}\right)^{q(\eta)} \frac{\left|\partial_{\mathbf{b}}^{k_{z}} F(z)\right|}{k_{z} ! L^{k_{z}}(z)} .
\end{gathered}
$$

Hence,

$$
\begin{gathered}
\quad \max \left\{\left|\partial_{\mathbf{b}}^{k_{z}} F(z+t \mathbf{b})\right|:|t| \leq \eta / L(z)\right\} \leq \\
\leq\left(2\left(\lambda_{\mathbf{b}}(\eta)\right)^{2 N}\right)^{q(\eta)} \frac{L^{k_{z}}\left(z+\widetilde{t_{z}} \mathbf{b}\right)}{L^{k_{z}}(z)}\left|\partial_{\mathbf{b}}^{k_{z}} F(z)\right| \leq \\
\leq\left(2\left(\lambda_{\mathbf{b}}(\eta)\right)^{2 N}\right)^{q(\eta)}\left(\lambda_{\mathbf{b}}(\eta)\right)^{N}\left|\partial_{\mathbf{b}}^{k_{z}} F(z)\right| \leq \\
\leq\left(2\left(\lambda_{\mathbf{b}}(\eta)\right)^{2 N}\right)^{q(\eta)}\left(\lambda_{\mathbf{b}}(\eta)\right)^{N}\left|\partial_{\mathbf{b}}^{k_{z}} F(z)\right| .
\end{gathered}
$$

Thus, we obtain (3) with $n_{0}=N_{\mathbf{b}}(F, L)$ and

$$
P_{1}(\eta)=\left(2\left(\lambda_{\mathbf{b}}(\eta)\right)^{2 N}\right)^{q(\eta)}\left(\lambda_{\mathbf{b}}(\eta)\right)^{N}>1
$$

Sufficiency. Suppose that for each $\eta>0$ there exist $n_{0}=n_{0}(\eta) \in \mathbb{Z}_{+}$and $P_{1}=P_{1}(\eta) \geq 1$ such that for every $z \in \mathbb{C}^{n}$ there exists $k_{0}=k_{0}(z) \in \mathbb{Z}_{+}, 0 \leq k_{0} \leq n_{0}$, for which inequality (3) holds. We choose $\eta>1$ and $j_{0} \in \mathbb{N}$ such that $P_{1} \leq \eta^{j_{0}}$. For given $z \in \mathbb{C}^{n}, k_{0}=k_{0}(z)$ and $j \geq j_{0}$ by Cauchy's formula for $F(z+t \mathbf{b})$ as a function of one variable $t$

$$
\partial_{\mathbf{b}}^{k_{0}+j} F(z)=\frac{j !}{2 \pi i} \int_{|t|=\eta / L(z)} \frac{\partial_{\mathbf{b}}^{k_{0}} F(z+t \mathbf{b})}{t^{j+1}} d t
$$

Therefore, in view of (3) we have

$$
\frac{\left|\partial_{\mathbf{b}}^{k_{0}+j} F(z)\right|}{j !} \leq \frac{L^{j}(z)}{\eta^{j}} \max \left\{\left|\partial_{\mathbf{b}}^{k_{0}} F(z+t \mathbf{b})\right|:|t|=\frac{\eta}{L(z)}\right\} \leq P_{1} \frac{L^{j}(z)}{\eta^{j}}\left|\partial_{\mathbf{b}}^{k_{0}} F(z)\right|,
$$

Hence, for all $j \geq j_{0}, z \in \mathbb{C}^{n}$

$$
\frac{\left|\partial_{\mathbf{b}}^{k_{0}+j} F(z)\right|}{\left(k_{0}+j\right) ! L^{k_{0}+j}(z)} \leq \frac{j ! k_{0} !}{\left(j+k_{0}\right) !} \frac{P_{1}}{\eta^{j}} \frac{\left|\partial_{\mathbf{b}}^{k_{0}} F(z)\right|}{k_{0} ! L^{k_{0}}(z)} \leq \eta^{j_{0}-j} \frac{\left|\partial_{\mathbf{b}}^{k_{0}} F(z)\right|}{k_{0} ! L^{k_{0}}(z)} \leq \frac{\left|\partial_{\mathbf{b}}^{k_{0}} F(z)\right|}{k_{0} ! L^{k_{0}}(z)} .
$$

Since $k_{0} \leq n_{0}$, the numbers $n_{0}=n_{0}(\eta)$ and $j_{0}=j_{0}(\eta)$ are independent of $z$ and $t_{0}$, this inequality means that a function $F$ has bounded $L$-index in the direction $\mathbf{b}$ and $N_{\mathbf{b}}(F, L) \leq n_{0}+j_{0}$. The proof of Theorem 4 is complete.

Theorem 4 implies the next proposition that describes the boundedness of $L$-index in direction for an equivalent function to $L$. Let $L^{*}(z)$ be a positive continuous function in $\mathbb{C}^{n}$. We denote $L \asymp L^{*}$, if for some $\theta_{1}, \theta_{2}, 0<\theta_{1} \leq \theta_{2}<+\infty$, and for all $z \in \mathbb{C}^{n}$ the following inequalities hold $\theta_{1} L(z) \leq L^{*}(z) \leq \theta_{2} L(z)$.

Proposition 5. Let $L \in Q_{\mathbf{b}^{\prime}}^{n} L \asymp L^{*}$. A function $F \in \widetilde{\mathcal{H}}_{\mathbf{b}}^{n}$ has bounded $L^{*}$-index in the direction $\mathbf{b}$ if and only if $F$ is of bounded L-index in the direction $\mathbf{b}$. 
Proof. First, it is not easy to check that the function $L^{*}$ also belongs to the class $Q_{\mathbf{b}}^{n}$. Let $N_{\mathbf{b}}\left(F, L^{*}\right)<+\infty$. Then by Theorem 4 for every $\eta^{*}>0$ there exist $n_{0}\left(\eta^{*}\right) \in \mathbb{Z}_{+}$and $P_{1}\left(\eta^{*}\right) \geq 1$ such that for every $z \in \mathbb{C}^{n}$ and some $k_{0}, 0 \leq k_{0} \leq n_{0}$, inequality (3) holds with $L^{*}$ and $\eta^{*}$ instead of $L$ and $\eta$. But the condition $L \asymp L^{*}$ means that for some $\theta_{1}, \theta_{2} \in \mathbb{R}_{+}, 0<\theta_{1} \leq \theta_{2}<+\infty$ and for all $z \in \mathbb{C}^{n}$ the double inequality holds $\theta_{1} L(z) \leq L^{*}(z) \leq \theta_{2} L(z)$. Taking $\eta *=\theta_{2} \eta$ we obtain

$$
\begin{gathered}
P_{1}\left|\partial_{\mathbf{b}}^{k_{0}} F(z)\right| \geq \max \left\{\left|\partial_{\mathbf{b}}^{k_{0}} F(z+t \mathbf{b})\right|:|t| \leq \eta^{*} / L^{*}(z)\right\} \geq \\
\geq \max \left\{\left|\partial_{\mathbf{b}}^{k_{0}} F(z+t \mathbf{b})\right|:|t| \leq \eta / L(z)\right\} .
\end{gathered}
$$

Thus, by Theorem 4 in view of arbitrariness of $\eta^{*}$ the function $F(z)$ has bounded $L$-index in the direction $\mathbf{b}$. We can obtain the converse proposition by replacing $L$ with $L^{*}$.

Please note that Proposition 5 can be slightly refined. The following proposition is easy deduced from (1).

Proposition 6. Let $L_{1}(z), L_{2}(z)$ be positive continuous functions, $F \in \widetilde{\mathcal{H}}_{\mathbf{b}}^{n}$ be a function of bounded $L_{1}$-index in the direction $\mathbf{b}$, for all $z \in \mathbb{C}^{n}$ the inequality $L_{1}(z) \leq L_{2}(z)$ holds. Then $N_{\mathbf{b}}\left(L_{2}, F\right) \leq N_{\mathbf{b}}\left(L_{1}, F\right)$.

Using Fricke's idea [20], we obtain modification of Theorem 4.

Theorem 5. Let $L \in Q_{\mathbf{b}}^{n}$. If there exist $\eta>0, n_{0}=n_{0}(\eta) \in \mathbb{Z}_{+}$and $P_{1}=P_{1}(\eta) \geq 1$ such that for all $z \in \mathbb{C}^{n}$ there exists $k_{0}=k_{0}(z) \in \mathbb{Z}_{+}, 0 \leq k_{0} \leq n_{0}$, for which the inequality holds

$$
\max \left\{\left|\partial_{\mathbf{b}}^{k_{0}} F(z+t \mathbf{b})\right|:|t| \leq \eta / L(z)\right\} \leq P_{1}\left|\partial_{\mathbf{b}}^{k_{0}} F(z)\right|,
$$

then the function $F \in \widetilde{\mathcal{H}}_{\mathbf{b}}^{n}$ has bounded L-index in the direction $\mathbf{b} \in \mathbb{C}^{n} \backslash\{\mathbf{0}\}$.

Proof. Our proof is based on the proof of appropriate theorem for entire functions of bounded $L$-index in direction [21].

Assume that there exist $\eta>0, n_{0}=n_{0}(\eta) \in \mathbb{Z}_{+}$and $P_{1}=P_{1}(\eta) \geq 1$ such that for every $z \in \mathbb{C}^{n}$ there exists $k_{0}=k_{0}(z) \in \mathbb{Z}_{+}, 0 \leq k_{0} \leq n_{0}$, for which

$$
\max \left\{\left|\partial_{\mathbf{b}}^{k_{0}} F(z+t \mathbf{b})\right|:|t| \leq \frac{\eta}{L(z)}\right\} \leq P_{1}\left|\partial_{\mathbf{b}}^{k_{0}} F(z)\right| \text {. }
$$

If $\eta>1$, then we choose $j_{0} \in \mathbb{N}$ such that $P_{1} \leq \eta^{j_{0}}$. And for $\eta \in(0 ; 1]$ we choose $j_{0} \in \mathbb{N}$ obeying the inequality $\frac{j_{0} ! k_{0} !}{\left(j_{0}+k_{0}\right) !} P_{1}<1$. This $j_{0}$ exists because

$$
\frac{j_{0} ! k_{0} !}{\left(j_{0}+k_{0}\right) !} P_{1}=\frac{k_{0} !}{\left(j_{0}+1\right)\left(j_{0}+2\right) \cdot \ldots \cdot\left(j_{0}+k_{0}\right)} P_{1} \rightarrow 0, j_{0} \rightarrow \infty .
$$

Applying Cauchy's formula to the function $F(z+t \mathbf{b})$ as function of complex variable $t$ for $j \geq j_{0}$ we obtain that for every $z \in \mathbb{C}^{n}$ there exists integer $k_{0}=k_{0}(z), 0 \leq k_{0} \leq n_{0}$, and

$$
\partial_{\mathbf{b}}^{k_{0}+j} F(z)=\frac{j !}{2 \pi i} \int_{|t|=\frac{\eta}{L(z)}} \frac{\partial_{\mathbf{b}}^{k_{0}} F(z+t \mathbf{b})}{t^{j+1}} d t
$$

Taking into account (11), one has

$$
\frac{\left|\partial_{\mathbf{b}}^{k_{0}+j} F(z)\right|}{j !} \leq \frac{L^{j}(z)}{\eta^{j}} \max \left\{\left|\partial_{\mathbf{b}}^{k_{0}} F(z+t \mathbf{b})\right|:|t|=\frac{\eta}{L(z)}\right\} \leq P_{1} \frac{L^{j}(z)}{\eta^{j}}\left|\partial_{\mathbf{b}}^{k_{0}} F(z)\right| .
$$


In view of choice $j_{0}$ for $\eta>1$ and for all $j \geq j_{0}$ we deduce

$$
\frac{\left|\partial_{\mathbf{b}}^{k_{0}+j} F(z)\right|}{\left(k_{0}+j\right) ! L^{k_{0}+j}(z)} \leq \frac{j ! k_{0} !}{\left(j+k_{0}\right) !} \frac{P_{1}}{\eta^{j}} \frac{\left|\partial_{\mathbf{b}}^{k_{0}} F(z)\right|}{k_{0} ! L^{k_{0}}\left(z+t_{0} \mathbf{b}\right)} \leq \eta^{j_{0}-j} \frac{\left|\partial_{\mathbf{b}}^{k_{0}} F(z)\right|}{k_{0} ! L^{k_{0}}(z)} \leq \frac{\left|\partial_{\mathbf{b}}^{k_{0}} F(z)\right|}{k_{0} ! L^{k_{0}}(z)} .
$$

Since $k_{0} \leq n_{0}$, the numbers $n_{0}=n_{0}(\eta)$ and $j_{0}=j_{0}(\eta)$ are independent of $z$, and $z \in \mathbb{C}^{n}$ is arbitrary, the last inequality means that the function $F$ is of bounded $L$-index in the direction $\mathbf{b}$ and $N_{\mathbf{b}}(F, L) \leq n_{0}+j_{0}$.

If $\eta \in(0,1)$, then (12) implies for all $j \geq j_{0}$

$$
\frac{\left|\partial_{\mathbf{b}}^{k_{0}+j} F(z)\right|}{\left(k_{0}+j\right) ! L^{k_{0}+j}(z)} \leq \frac{j ! k_{0} ! P_{1}}{\left(j+k_{0}\right) !} \frac{\left|\partial_{\mathbf{b}}^{k_{0}} F(z)\right|}{\eta^{j} k_{0} ! L^{k_{0}}(z)} \leq \frac{\left|\partial_{\mathbf{b}}^{k_{0}} F(z)\right|}{\eta^{j} k_{0} ! L^{k_{0}}(z)}
$$

or in view of the choice of $j_{0}$

$$
\frac{\left|\partial_{\mathbf{b}}^{k_{0}+j} F(z)\right|}{\left(k_{0}+j\right) !} \frac{\eta^{k_{0}+j}}{L^{k_{0}+j}(z)} \leq \frac{\left|\partial_{\mathbf{b}}^{k_{0}} F(z)\right|}{k_{0} !} \frac{\eta^{k_{0}}}{L^{k_{0}}(z)} .
$$

Thus, the function $F$ has bounded $\tilde{L}$-index in the direction $\mathbf{b}$, where $\tilde{L}(z)=\frac{L(z)}{\eta}$. Then by Proposition 5 the function $F$ is of bounded $L$-index in the direction $\mathbf{b}$. Theorem is proved.

\section{Bounded Index in Direction in Bounded Domain}

Let $D$ be a bounded domain in $\mathbb{C}^{n}$. If inequality (1) is fulfilled for all $z \in D$ instead $\mathbb{C}^{n}$, then $F$ is called function of bounded L-index in the direction $\mathbf{b}$ in the domain $D$. The least such integer $m_{0}$ is called the $L$-index in the direction $\mathbf{b}$ in the domain $D$ and is denoted by $N_{\mathbf{b}}(F, L, D)=m_{0}$. By $\bar{D}$ we denote a closure of domain $D$.

Theorem 6. Let $D$ be an arbitrary bounded domain in $\mathbb{C}^{n}, \mathbf{b} \in \mathbb{C}^{n} \backslash\{\mathbf{0}\}$ be arbitrary direction. If $L: \mathbb{C}^{n} \rightarrow$ $\mathbb{R}_{+}$is continuous function, $F \in \mathcal{H}_{\mathbf{b}}^{n}$ and $(\forall p \in \mathbb{N}) \partial_{\mathbf{b}}^{p} F \in \mathcal{H}_{\mathbf{b}}^{n}$ and $\left(\forall z^{0} \in \bar{D}\right): \quad F\left(z^{0}+t \mathbf{b}\right) \not \equiv 0$, then $N_{\mathbf{b}}(F, L, \bar{D})<\infty$.

Proof. Proof of this theorem is similar to proof of corresponding lemma in [13]. For every given $z^{0} \in \bar{D}$ we develop the entire function $F\left(z^{0}+t \mathbf{b}\right)$ in power series by powers $t$

$$
F\left(z^{0}+t \mathbf{b}\right)=\sum_{m=0}^{\infty} \frac{\partial_{\mathbf{b}}^{m} F\left(z^{0}\right)}{m !} t^{m}
$$

in the disc $\left\{t \in \mathbb{C}:|t| \leq \frac{1}{L\left(z^{0}\right)}\right\}$.

The quantity $\frac{\left|\partial_{\mathbf{b}}^{m} F\left(z^{0}\right)\right|}{m !}$ is the modulus of coefficient of power series (13) at the point $t=0$. Substitute $t=\frac{1}{L\left(z^{0}\right)}$. Since $F \in \mathcal{H}_{\mathbf{b}^{\prime}}^{n}$ for every $z_{0} \in \bar{D}$

$$
\frac{\left|\partial_{\mathbf{b}}^{m} F\left(z^{0}\right)\right|}{m ! L^{m}\left(z^{0}\right)} \rightarrow 0 \quad(m \rightarrow \infty),
$$

i.e., there exists $m_{0}=m\left(z^{0}, \mathbf{b}\right)$ such that inequality (1) holds at the point $z=z^{0}$ for all $m \in \mathbb{Z}_{+}$.

We will show that $\sup \left\{m_{0}: z^{0} \in \bar{D}\right\}<+\infty$. On the contrary, we suppose that the set of all values $m_{0}$ is unbounded in $z^{0}$, that is $\sup \left\{m_{0}: z^{0} \in \bar{D}\right\}=+\infty$. Hence, for every $m \in \mathbb{Z}_{+}$there exists $z^{(m)} \in \bar{D}$ and $p_{m}>m$

$$
\frac{\left|\partial_{\mathbf{b}}^{p_{m}} F\left(z^{(m)}\right)\right|}{p_{m} ! L^{p_{m}}\left(z^{(m)}\right)}>\max \left\{\frac{\left|\partial_{\mathbf{b}}^{k} F\left(z^{(m)}\right)\right|}{k ! L^{k}\left(z^{(m)}\right)}: 0 \leq k \leq m\right\} .
$$


Since $\left\{z^{(m)}\right\} \subset \bar{D}$, there exists subsequence $z^{\prime(m)} \rightarrow z^{\prime} \in \bar{G}$ as $m \rightarrow+\infty$. By Cauchy's formula one has

$$
\frac{\partial_{\mathbf{b}}^{p} F(z)}{p !}=\frac{1}{2 \pi i} \int_{|t|=r} \frac{F(z+t \mathbf{b})}{t^{p+1}} d t
$$

for any $p \in \mathbb{N}, z \in D$. Rewrite (14) in the form

$$
\begin{gathered}
\max \left\{\frac{\left|\partial_{\mathbf{b}}^{k} F\left(z^{(m)}\right)\right|}{k ! L^{k}\left(z^{(m)}\right)}: 0 \leq k \leq m\right\}< \\
<\frac{1}{L^{p^{m}}\left(z^{(m)}\right)} \int_{|t|=r / L\left(z^{(m)}\right)} \frac{\left|F\left(z^{(m)}+\mathbf{b}\right)\right|}{|t|^{p^{m+1}}}|d t| \leq \frac{1}{r^{m m}} \max \left\{|F(z)|: z \in D_{r}\right\},
\end{gathered}
$$

where $D_{r}=\bigcup_{z^{*} \in \bar{D}}\left\{z \in \mathbb{C}^{n}:\left|z-z^{*}\right| \leq \frac{|b| r}{L\left(z^{*}\right)}\right\}$. We can choose $r>1$, because $F \in \mathcal{H}_{\mathbf{b}}^{n}$. Evaluating limit for every directional derivative of fixed order in (15) as $m \rightarrow \infty$ we obtain

$$
\left(\forall k \in \mathbb{Z}_{+}\right): \quad \frac{\left|\partial_{\mathbf{b}}^{k} F\left(z^{\prime}\right)\right|}{k ! L^{k}\left(z^{\prime}\right)} \leq \varlimsup_{m \rightarrow \infty} \frac{1}{r^{p_{m}}} \max \left\{|F(z)|: z \in D_{r}\right\} \leq 0 .
$$

The passing to the limit is possible because $\partial_{\mathbf{b}}^{k} F$ is joint continuous. Thus, all derivatives in the direction $\mathbf{b}$ of the function $F$ at the point $z^{\prime}$ equal 0 and $F\left(z^{\prime}\right)=0$. In view of (13) $F\left(z^{\prime}+t \mathbf{b}\right) \equiv 0$. This is a contradiction.

\section{Conclusions}

The proposed approach can be applied in analytic theory of differential equations. It is known that concept of bounded index allows the investigation of properties of analytic solutions of linear higher-order differential equations with analytic coefficients. Therefore, it leads to the question of what the additional conditions are, providing index boundedness of every slice holomorphic solutions for linear higher-order directional derivative equations with slice holomorphic coefficients? In other words, is joint continuity a sufficient condition?

Since there are known analogs of Cauchy's formula for quaternionic variables and for Clifford algebras, the authors assume that The results in this paper can be generalized in these cases, i.e., in the case of slice holomorphic functions of quaternionic variable.

Author Contributions: These authors contributed equally to this work.

Funding: This research received no external funding.

Conflicts of Interest: The authors declare no conflict of interest.

\section{References}

1. Bandura, A.; Skaskiv, O. Asymptotic estimates of entire functions of bounded L-index in joint variables. Novi Sad J. Math. 2018, 48, 103-116. [CrossRef]

2. Nuray, F.; Patterson, R.F. Multivalence of bivariate functions of bounded index. Le Matematiche 2015, 70, 225-233. [CrossRef]

3. Nuray, F.; Patterson, R.F. Vector-valued bivariate entire functions of bounded index satisfying a system of differential equations. Mat. Stud. 2018, 49, 67-74. [CrossRef]

4. Bandura, A.; Petrechko, N.; Skaskiv, O. Maximum modulus in a bidisc of analytic functions of bounded L-index and an analogue of Hayman's theorem. Mat. Bohem. 2018, 143, 339-354. [CrossRef]

5. Bandura, A.; Skaskiv, O. Sufficient conditions of boundedness of L-index and analog of Hayman's Theorem for analytic functions in a ball. Stud. Univ. Babeş-Bolyai Math. 2018, 63, 483-501. [CrossRef]

6. Bandura, A.I.; Skaskiv, O.B.; Tsvigun, V.L. Some characteristic properties of analytic functions in $\mathbb{D} \times \mathbb{C}$ of bounded L-index in joint variables. Bukovyn. Mat. Zh. 2018, 6, 21-31.

7. Bandura, A.I.; Skaskiv, O.B. Entire functions of bounded L-index in direction. Mat. Stud. 2007, 27, 30-52. (In Ukrainian) 
8. Kuzyk, A.D.; Sheremeta, M.N. Entire functions of bounded l-distribution of values. Math. Notes 1986, 39, 3-8. [CrossRef]

9. Kuzyk, A.D.; Sheremeta, M.N. On entire functions, satisfying linear differential equations. Differ. Equ. 1990, $26,1716-1722$.

10. Lepson, B. Differential equations of infinite order, hyperdirichlet series and entire functions of bounded index. Proc. Symp. Pure Math. 1968, 2, 298-307.

11. Macdonnell, J.J. Some Convergence Theorems for Dirichlet-Type Series Whose Coefficients are Entire Functions of Bounded Index. Ph.D. Thesis, Catholic University of America, Washington, DC, USA, 1957.

12. Strelitz, S. Asymptotic properties of entire transcendental solutions of algebraic differential equations. Contemp. Math. 1983, 25, 171. [CrossRef]

13. Bandura, A.I. Sum of entire functions of bounded L-index in direction. Mat. Stud. 2016, 45, 149-158. [CrossRef]

14. Kosanyak, M. Slice Entire Functions of Bounded Index in Direction, Master's Thesis, Ivan Franko National University of Lviv, Lviv, Ukraine, 2018.

15. Sheremeta, M. Analytic Functions of Bounded Index; VNTL Publishers: Lviv, Ukraine, 1999; 141p.

16. Bandura, A.I.; Skaskiv, O.B. Sufficient sets for boundedness $L$-index in direction for entire functions. Mat. Stud. 2008, 30, 177-182.

17. Fricke, G.H. Functions of bounded index and their logarithmic derivatives. Math. Ann. 1973, 206, $215-223$. [CrossRef]

18. Sheremeta, M.N.; Kuzyk, A.D. Logarithmic derivative and zeros of an entire function of bounded $l$-index. Sib. Math. J. 1992, 33, 304-312. [CrossRef]

19. Kushnir, V.O.; Sheremeta, M.M. Analytic functions of bounded l-index. Mat. Stud. 1999, 12, 59-66.

20. Fricke, G.H. Entire functions of locally slow growth. J. Anal. Math. 1975, 28, 101-122. [CrossRef]

21. Bandura, A.I. A modified criterion of boundedness of $L$-index in direction. Mat. Stud. 2013, 39, 99-102.

(C) 2019 by the authors. Licensee MDPI, Basel, Switzerland. This article is an open access article distributed under the terms and conditions of the Creative Commons Attribution (CC BY) license (http://creativecommons.org/licenses/by/4.0/). 